\title{
Developing an Influenza Vaccine Based on Genetic Engineering: The Use of Enhanced Green Fluorescent Protein to Increase the Selection of Recombinant Baculovirus with Genes Encoding Influenza Viral-like Particles
}

\section{Fera Ibrahim ${ }^{1,2}$, Silvia Tri Widyaningtyas ${ }^{2}$, Debbie Rizqoh ${ }^{3}$, and Budiman Bela ${ }^{1,2}$}

${ }^{1}$ Department of Clinical Microbiology, Faculty of Medicine, Universitas Indonesia - Dr. Cipto Mangunkusumo General Hospital, Jakarta, Indonesia

${ }^{2}$ Virology and Cancer Pathobiology Research Center, Faculty of Medicine, Universitas Indonesia - Dr Ciptomangunkusumo General Hospital, Jakarta, Indonesia

${ }^{3}$ Magister Program of Biomedical Science, Faculty of Medicine, Universitas Indonesia, Jakarta, Indonesia

Corresponding Author:

Fera Ibrahim

feraib@yahoo.fr

Received: 23 February 2019

Accepted: 6 March 2019

Published: 25 March 2019

Publishing services provided by

Knowledge E

(c) Fera Ibrahim et al. This article is distributed under the terms of the Creative Commons

Attribution License, which permits unrestricted use and redistribution provided that the original author and source are credited.

Selection and Peer-review under the responsibility of the ICO-HELICS Conference Committee.

\section{Abstract}

Developing vaccines based on viral-like particles (VLPS) can be beneficial. VLPs represent viral antigens that resemble viruses in nature. Unlike viruses that can replicate inside their host causing infection, VLPs have no capability to replicate and cause infection due to the lack of a viral genome. These properties make VLPs a preferable vaccine rather than inactivated viruses. In this research, VLPs were produced using a baculovirus infection system. Upon infection in SF9 insect cells, recombinant baculovirus produces new baculovirus proteins as well as the influenza proteins hemagglutinin (HA), neuraminidase (NA), and matrix (M1) protein, which can auto assemble into VLPs. VLPs can be separated from baculovirus using an ultracentrifuge. The first step of VLP production is selection of the baculovirus with recombinant genes encoding influenza proteins. The obstacle in selecting recombinant baculovirus is that infected SF9 cells do not produce specific cytopathogenic effects. To assist in the observation of baculovirus production in SF9 cells, we developed a protein reporter system for recombinant baculovirus production. Enhanced green fluorescent protein (eGFP) was used as a reporter protein. The gene encoding eGFP was cloned into the same transient plasmid that also encodes HA, NA, and matrix protein M1. Those proteins were expressed under four different open reading frames. This plasmid was co-transfected with a linearized baculovirus genome. Structural proteins of baculovirus and VLP forming recombinant proteins as well as the reporter protein were produced. Structural proteins of the baculovirus together with the genome of the baculoviruscontaining recombinant genes would co-assemble, producing recombinant baculovirus. The recombinant proteins then assembled, producing VLPs, and expression of the reporter protein eGFP would be visible in the cytoplasm by fluorescence microscopy. Using a TALI cytometer, the percentage of cells that produced GFP was determined to be $3 \%$ of the total population, indicating that eGFP can be used to observe the production of recombinant baculovirus in SF9 cells. The infectivity of baculovirus 
can be improved by passaging baculovirus in SF9 cells and isolating highly infective recombinant baculovirus by plaque assays.

Keywords: VLP, Recombinant baculovirus, eGFP

\section{Introduction}

Although poultry is an $\mathrm{H} 5 \mathrm{~N} 1$ influenza virus host, this virus can also infect humans with serious manifestation. Human can be infected by $\mathrm{H} 5 \mathrm{~N} 1$ by contact with poultry or their products. Evidence of human-to-human infection does not exist except in 'family cluster' cases. The presence of H5N1 infection in poultry and various animals needs to be watched out. This is based on the ability of the H5N1 virus genome to recombine and undergo spontaneous mutation, which can alter the virus profile, allowing it to adapt to horizontal infection [1].

As the chances of human-to-human infection of new strains of H5N1 have increased, vaccine development is ongoing. One of the H5N1 vaccines developed is a viral-like particle (VLP). VLPs have a virus-like structure without the viral genome. This influenza VLP consists of virus surface glycoprotein, hemagglutinin (HA), neuraminidase (NA), and matrix protein M1. As a multiprotein vaccine, VLPs have advantages compared to subunit or inactivated vaccines. VLPs has a virus-like immunogenicity because the antigens carried have the same antigenic structure as viruses. It is also considered safer because it does not contain genetic material. The influenza virus has the ability to recombine genomes with other influenza virus subtypes. This recombination will produce new viruses with new characteristics [2]. Based on this theory, VLPs can be helpful in preventing recombinant viruses with a new genome.

VLPs are made by cloning VLP genes into a transient plasmid. The protein-coding genes were cloned into one plasmid type in which each protein is expressed under p10 promoter and terminator. Following this, the transient plasmid is co-transfected into SF9 insect cells along with the linearized baculovirus genome. In SF9 cells, the transient plasmid and linearized baculovirus genome recombine to produce a full baculovirus genome that produces recombinant baculovirus that contains recombinant protein-producing genes [3].

There are some issues with the VLP-based vaccine production technique, such as the observation of baculovirus-infected cells because damaged cells are not evident. In this 
research, an enchanted green fluorescent protein (eGFP) was added to better visualize recombinant baculovirus. eGFP was expressed separately from the VLP proteins. eGFP do not encode transmembrane proteins or contain other sequence signals for export to the outer cell membrane. With this profile, the chance of eGFP remaining on the SF9 cell lipid bilayer membrane is low, and thus, the expression of eGFP is not linked to VLP.

\section{Material and Method}

\section{1. pBAcH5N1MeGFP plasmid pBAcH5N1MeGFP plasmid}

The transient plasmid consisted of VLP-protein coding genes, including HA, NA, M1, and eGFP. Each plasmid was expressed under a p10 promoter.

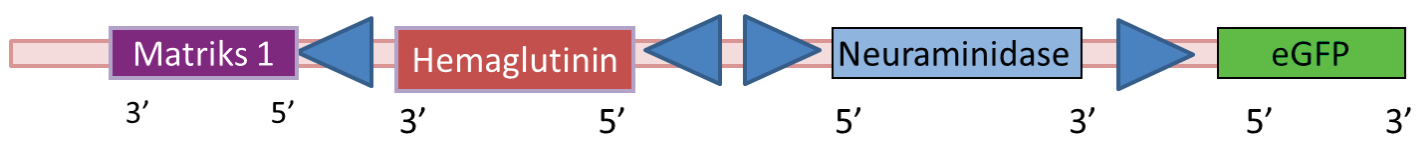

Figure 1: Virus-like particle (VLP)-protein coding gene position. VLP and marker on pBacGus 4x. Head of the arrow: p10 promoter. eGFP: enhanced green fluorescent protein.

\subsection{Baculogold and recombinant transient vector coinfection}

Baculogold is the linear state of baculovirus DNA. It contains lethal genes and is unable to produce baculovirus. Baculogold co-transfection with the transient plasmid recombinant gene carrier resulted in homologous recombination between Baculogold and the transient vector, with a recombination frequency of approximately $99 \%$ in insect cells. This recombination caused the Baculogold DNA to acquire genes critical for virus life. Recombination was performed as follows: before transfection, $2 \mathrm{~mL}$ of SF9 cells $\left(5 \times 10^{5}\right.$ cells $\left./ \mathrm{mL}\right)$ were plated on a 6 -well plate and allowed to adhere. Transfection reagent was prepared as follows: Mixture 1, 100 ng Bac Vector 1000 (Novagen) mixed with 500 ng transient plasmid with HA, NA, M1, and eGFP coding genes. SFIII medium without serum were added until a volume of $25 \mu$ Lwas reached; Mixture 2, $5 \mu \mathrm{L}$ of Insect Genejuce Transfection Reagent 1000 (Novagen) was added into $20 \mu \mathrm{L}$ of nuclease-free water (Ambion). After that, mixture 1 was added to mixture 2 and incubated 15 min at room temperature. During the incubation, cells were washed with sterile phosphatebuffered saline (PBS). Then, $950 \mu \mathrm{L} \mathrm{SFIII} \mathrm{medium} \mathrm{without} \mathrm{serum} \mathrm{(Invitrogen)} \mathrm{was} \mathrm{added}$ into mixture 1 and 2 , which was then added to SF9 cells. After $1 \mathrm{~h}$ at room temperature, $2 \mathrm{~mL}$ of media was added. After 4 days at room temperature, SF9 cells were infected 
with the culture supernatants. The remainders of the supernatants were stored at $4^{\circ} \mathrm{C}$ or $-80^{\circ} \mathrm{C}$.

\subsection{Recombinant baculovirus infection in SF9 cells}

SF9 cells were plated on $3 \mathrm{~cm}$ diameter plates until confluent. Supernatants containing the virus were diluted 1:100 and $100 \mu \mathrm{L}$ was added to monolayer SF9 cells. Infections were performed for 1 hour and every 15 min cells were shaken to ensure the virus was spread evenly. Following infection, supernatants were removed and new cell media was added. Cells were incubated at room temperature for 3-5 days. To increase virus infectivity, viral passages were performed. Cells were observed with a light and fluorescence microscope. Fluorescence indicated that the cell had been infected with recombinant baculovirus.

\subsection{Tali $^{T M}$ image-based cytometer}

Cells were diluted with PBS to a concentration of $5 \times 10^{5}$ cells $/ \mathrm{mL}$ and the number of fluorescent cells was counted. Then, $25 \mu \mathrm{L}$ of diluted cells were placed on a Tali slide (Invitrogen), which was inserted into Tali ${ }^{T M}$ image-based cytometer (Invitrogen).

\section{Result and Discussion}

Naturally, baculovirus is a virus that attacks Autographa california (Ac), an alfalfa looper. Baculovirus is a supercoiled circular double-stranded DNA virus. This genome ranges in size from 80 to more than $180 \mathrm{~kb}$ and encodes approximately 90-180 genes. This rod-shaped virus is $200 \mu \mathrm{m}$ long and has a diameter of $30 \mu \mathrm{m}$.

Baculovirus has a biphasic life cycle and produces two different phenotypes of virus progeny: occlusion-derived virions (ODVs) and budded virions (BVs) [4]. ODVs are derived from occlusion bodies (OBs) and virions inside OBs are called occluded virions (ODs). BVs originate from virus cell budding. These two virion types differ in their envelopes and in their ability to infect cells. ODVs or ODs are needed to infect one larva from another. On the other hand, BVs are needed to infect cultured cells. ODs are generated from the larva's body and have been prepared to face external environmental conditions. The existence of ODs in OVs will make ODs resistant to the physical, biological, and chemical environment. OBs can allow for the survival of ODs 
for many years in the external environment and do not lose their infectious properties [5].

Following three viral passages, recombinant baculovirus-infected cells showed some altered morphologies, including the formation of inclusion bodies and multinucleated giant cells; cell shape became square; cell clumping; and enlargement. Cell debris was also observed.
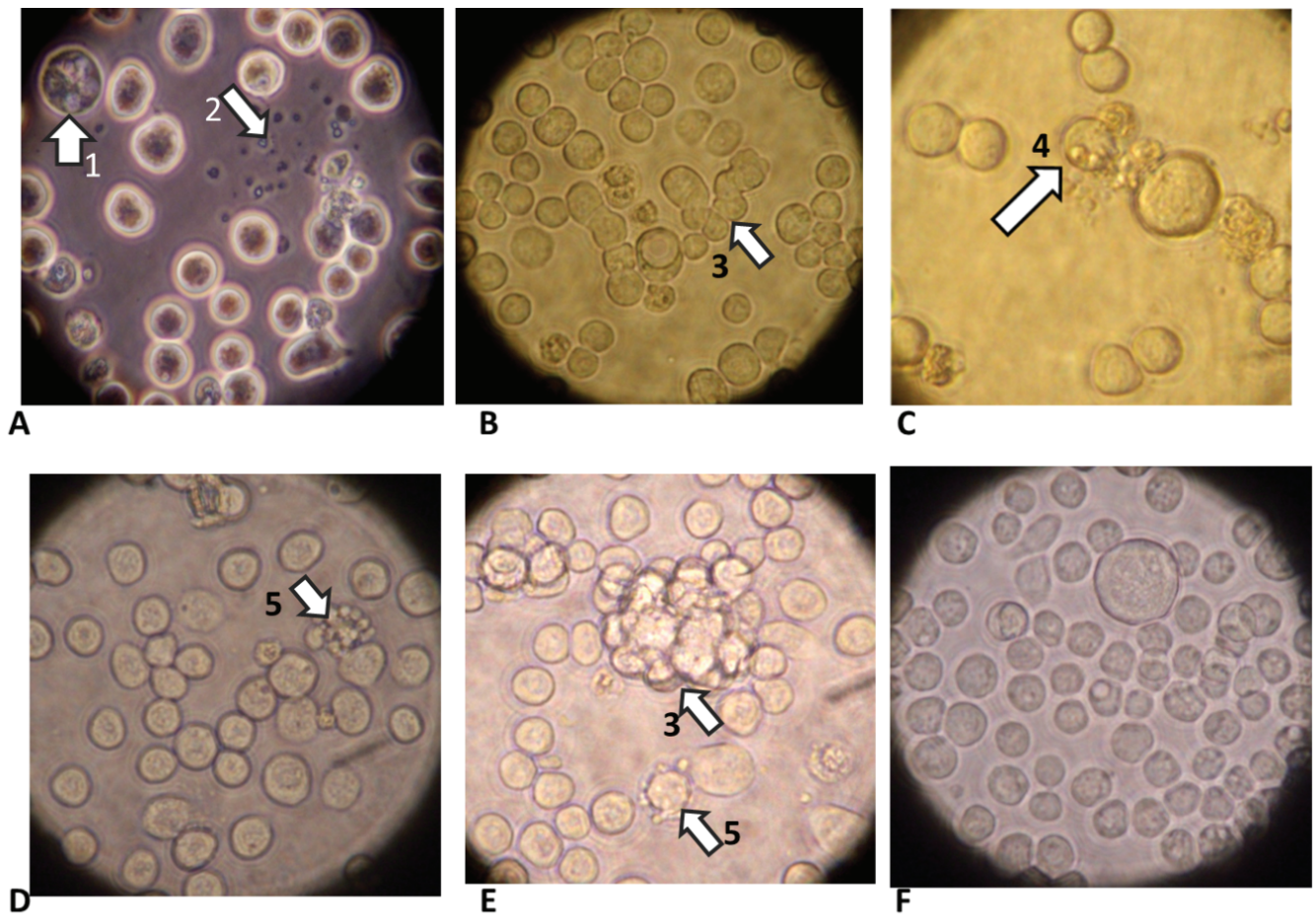

Figure 2: Morphological changes of recombinant baculovirus-infected cells, observed with a light microscope. (A-E) multinucleated cell (arrow 1), cell debris (arrow 2), clumping cell (arrow 3), inclusion bodies (arrow 4), and bumps on the cell membrane (arrow 5). None of the morphological changes were observed in normal cells $(F)$.

The morphological changes of SF9 cells indicated that the recombinant baculovirus affected these cells. Baculovirus-infected cells will undergo fusion [6]. Fusion of infected cells results in multinucleated and clumping cells. Baculovirus infection will also induce cells to enter a resting $\mathrm{G} 2 / \mathrm{M}$ phase, resulting in cell death [7]. In general, the disruption of cell cycle will result in apoptosis, which is evident morphologically by cell swelling. Following this, protrusions are formed on the cell membrane. After swelling to a certain volume, cells are fragmented into smaller sizes [8]. This apoptotic phenomenon was also observed in recombinant baculovirus infected-cells (Figure 2).

The addition of the marker protein eGFP allowed for the differentiation of infected recombinant baculovirus cells that produce recombinant proteins carried by the baculovirus. Some studies have investigated the use of eGFP as a baculovirus marker [9]. 
In one study, eGFP was expressed as a baculovirus structural protein. The addition of eGFP has been shown to help in the plaque selection process, which was performed using UV light at $365 \mathrm{~nm}$. In this research, eGFP was expressed separately, not as part of the baculovirus structural or VLP proteins. On the third viral passage, approximately $3 \%$ of cells were eGFP-positive (Figure 3).

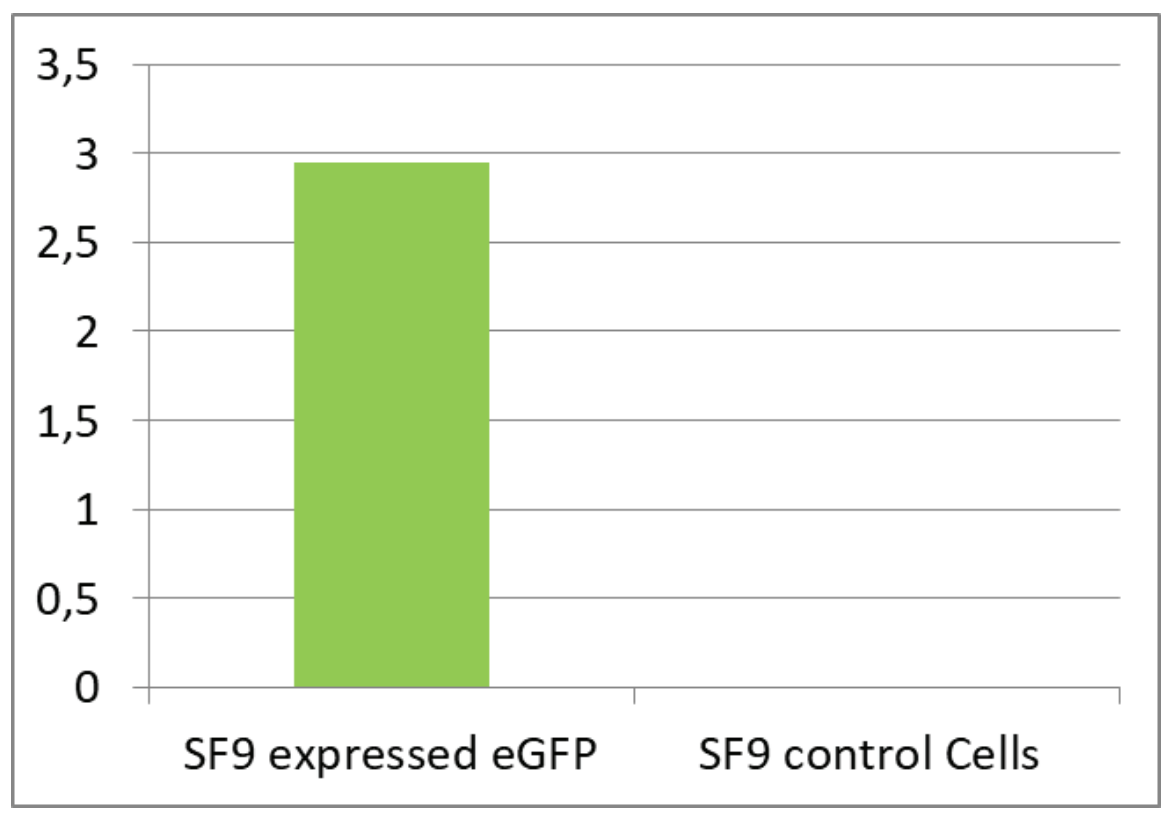

Figure 3: Percentage of eGFP-expressing cells. 1, SF9 cells infected with the third baculovirus passage; 2 , SF9 cell control.

Based on fluorescence microscopy, eGFP fluorescence was not high in intensity (Figure 4); the intensity was correlated with the molecular amount in cells.

On the fourth passage, an increased number of fluorescent cells were observed (Figure 5), indicating that more eGFP was expressed. This is hypothesized to be caused by the recombinant baculovirus, which can produce the recombinant protein, eGFP, with high intensity.

Thus, recombinant baculovirus was produced, but the capacity to infect was still low, as indicated by the percentage of fluorescent cells after the third passage. The success of recombinant baculovirus formation is dependent on the ability of the transient plasmid to recombine with the linearized baculovirus genome. This recombination occurs in SF9 cells, producing baculovirus structural proteins and the recombinant baculovirus genome.

To obtain infectious baculovirus, the plaque test must be performed to screen recombinant baculovirus, which has a high infectious ability. In this test, virus isolation from the plaque is performed. One plaque represents one virus. Each plaque is tested in terms of recombinant protein producing ability. SDS-PAGE and western blotting can be 


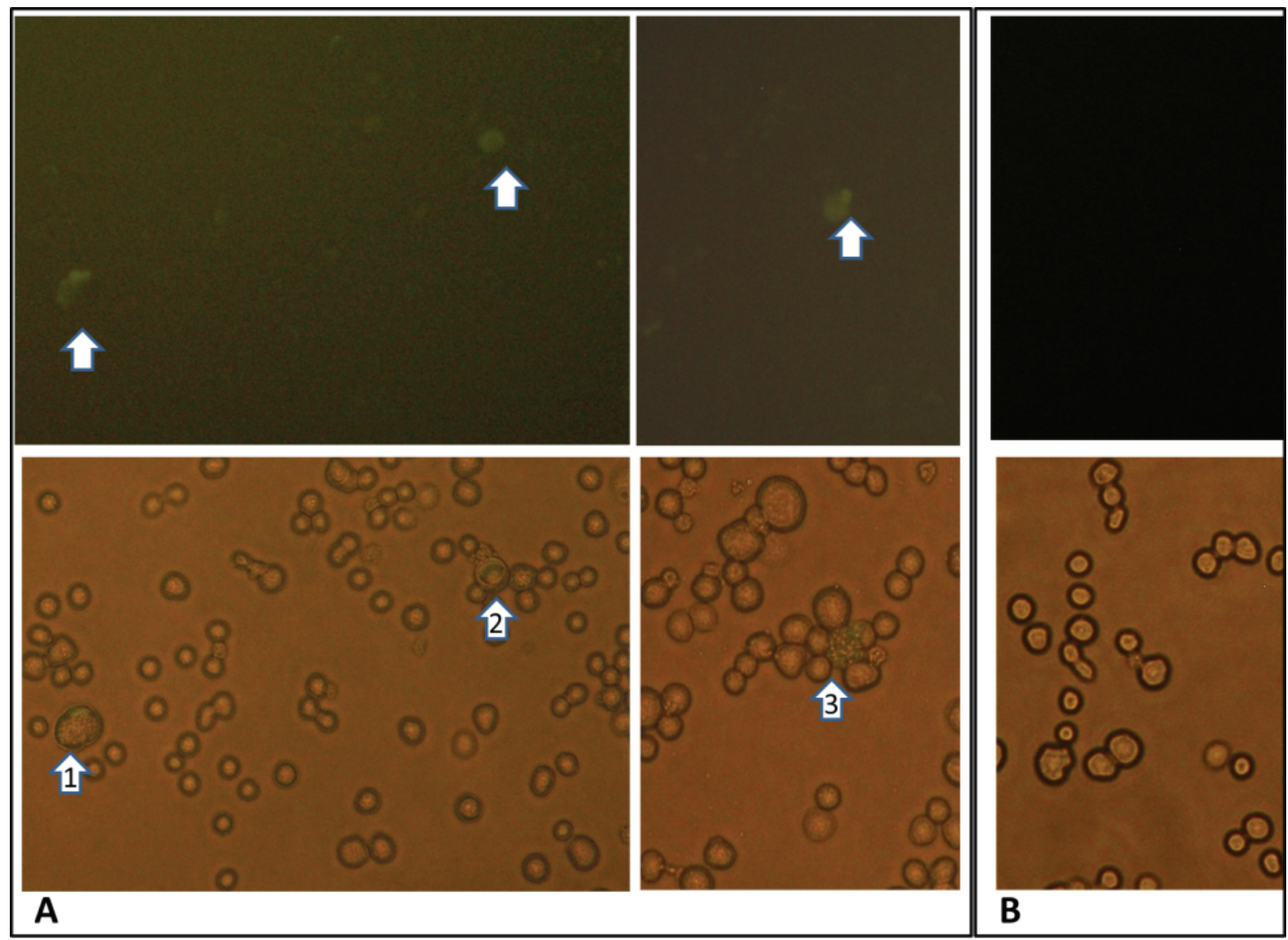

Figure 4: Observation of eGFP by fluorescence microscopy. (A) Third passage of recombinant baculovirusinfected cells. (B) Control.

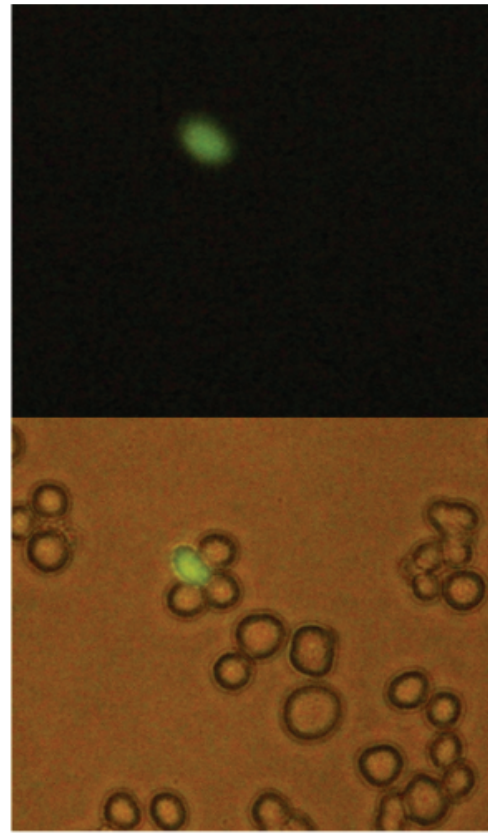

A

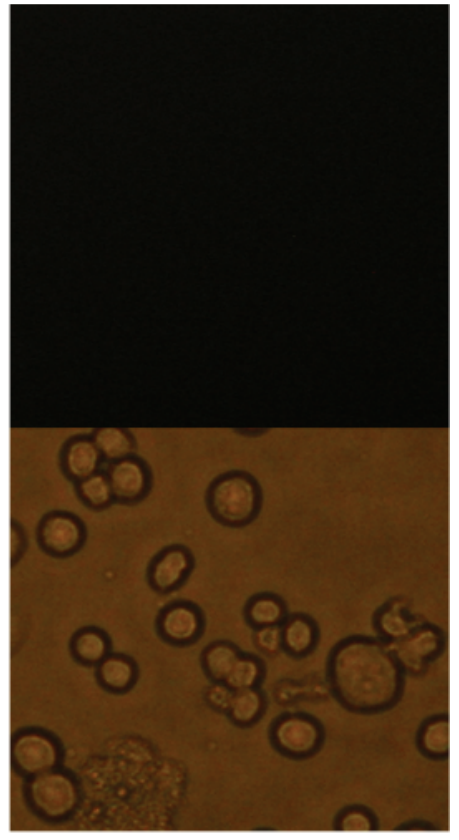

B

Figure 5: Observation of eGFP by fluorescence microscopy. (A) Fourth passage of recombinant baculovirusinfected cells. (B) Control. 
used to analyze recombinant proteins, and PCR can be performed to determine if the recombinant baculovirus is present.

\section{Acknowledgments}

This research is part of the research entitled "Development of Pandemic Influenza Vaccine based on Genetic Engineering" (RT-2014-837), which was funded by the Ministry of Research, Technology, and Higher Education on "Insentif Riset Sistem Inovasi Nasional".

\section{Conflict of Interest}

The authors declare no conflicts of interest

\section{References}

[1] Khurana S,Wu J, Erma N, Verma S, Raghunandan R,Manischewitz J, King LR, Kpamegan E, Pincus S, Smith G, Gregory Glenn and Golding H 2011 J.Virol. 85 1094554

[2] Boni MF, de Jong MD, van Doorn HR and Holmes EC 2010 PLoS One 03 e10434

[3] Kost TA, Condreay JP and Jarvis DL 2005 Nat Biotechnol 23 567-75

[4] Hu Z, Luijckx T, van Dinten LC, van Oers MM, Hajós JP, Bianchi FJ, van Lent JW, Zuidema D, Vlak JM 1999 J Gen Virol 80 1045-53

[5] Rohrmann GF 2013 Baculovirus Molecular Biology [Internet] ${ }^{\text {rd }}$ Ed pg 17-32

[6] latrou K and Swevers L 2005 J.Biotechnology 120 237-50

[7] Braunagel SC, Parr R, Belyavskyi M, and Summers MD 1998 Virology 244 195-211

[8] Elmore S 2007 Toxicol Pathol 35 495-516

[9] Wilson LE, Wilkinson N, Marlow SA, Possee RD and King LA 1997 Biotechniques 22 674-6, 678-81 